\title{
Aptamer-lipid nanoparticle conjugates for RNAi in bone
}

RNA interference (RNAi)based bone anabolic therapies are promising strategies for treating skeletal disorders such as osteoporosis; however, a lack of efficient osteoblast-specific delivery methods has hampered the implementation of such therapies in the clinical setting. New research published in Nature Medicine has demonstrated osteoblast-specific uptake of aptamer-functionalized lipid nanoparticles (LNPs) containing osteogenic small interfering RNA (siRNA) molecules. This advance might facilitate the clinical translation of RNAi-based treatments for bone disorders.

Senior investigator Ge Zhang and colleagues had previously developed a system that delivered therapeutic siRNA molecules to osteoblast-enriched boneformation surfaces on the basis of preferential binding to lowly crystallized hydroxyapatite and amorphous calcium phosphonate. Although effective, the therapeutic utility of the system was marred by concerns of potentially harmful off-target effects, which prompted development of a truly osteoblast-specific delivery system.

"Aptamers are single-stranded oligonucleotides that use distinct tertiary structures to specifically bind to target cells," explains Zhang. In their new study, the researchers initially screened a library of $10^{15}$ single-stranded DNA molecules to identify aptamers that specifically bind to the surface of primary rat osteoblasts and not to other cell types, in particular rat osteoclasts. Two aptamers identified in the screen also bound specifically to human osteoblasts; $\mathrm{CH} 6$ was selected for functional testing on the basis of its secondary structure and short sequence length.

The specificity and efficiency of the CH6-conjugated LNPs

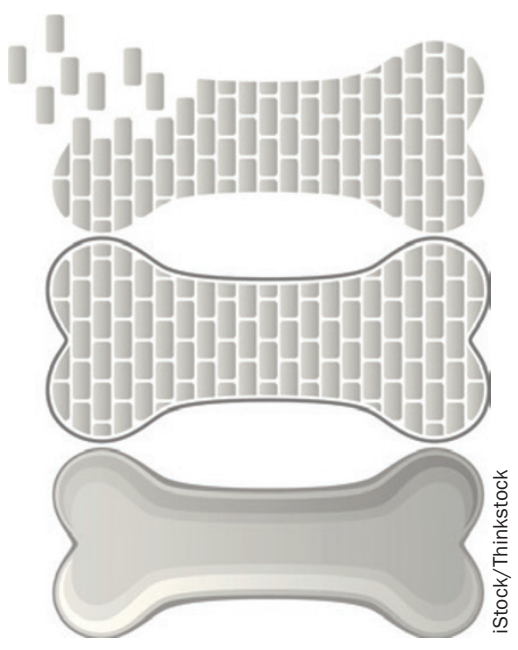

was tested using fluorescently labelled Plekho1 siRNAs, which target the gene that encodes the intracelullar negative regulator of bone formation pleckstrin homology domain-containing family $\mathrm{O}$ member 1 . In cultured rat osteoblasts, the efficiency of gene knockdown with CH6LNP-siRNAs was significantly higher than that of siRNAs delivered in either unconjugated LNPs or LNPs conjugated to a random aptamer.

In vivo administration of CH6-LNP-siRNAs resulted in colocalization of the siRNAs with cellular markers of osteoblasts and a reduction of levels of Plekho1 mRNA transcripts that did not occur in osteoclasts or other tissues such as the liver. Furthermore, administration of CH6-LNPsiRNAs to ovariectomized rats led to increased bone anabolism compared with administration of siRNAs delivered in LNPs that were either unconjugated or linked to the random aptamer.

\section{Jennifer Sargent}

Original article Liang, C. et al. Aptamerfunctionalized lipid nanoparticles targeting osteoblasts as a novel RNA interferencebased bone anabolic strategy. Nat. Med. doi:10.1038/nm.3791 